\section{Prescripción de fármacos bloqueadores del sistema renina angiotensina en pacientes con enfermedad renal crónica etapa 3 en atención primaria de salud}

\author{
MARCEL ALVAREZ ${ }^{1}$, LEOPOLDO ARDILES ${ }^{1}$
}

\section{Prescription of renin-angiotensin-aldosterone system blockers in patients with stage 3 chronic kidney disease}

Background: To reduce the progression of chronic kidney disease (CKD) and cardiovascular risk, the guidelines recommend the blockade of the renin-angiotensin-aldosterone system (RAAS) in patients with proteinuria. Aim: To assess the frequency of enalapril or losartan use in diabetics or hypertensive patients with stage 3 CKD. Material and Methods: Review of clinical records of patients with CKD in an urban primary care clinic. Results: We identified 408 subjects aged 40 to 98 years (66\% women) with stage 3 CKD. Sixty six percent had only hypertension and 34\% were diabetic with or without hypertension. Seventy four percent received RAAS blockers (52\% used enalapril, 45\% losartan and $2 \%$ both medications). RAAS blockers were used in $70 \%$ of hypertensive and $78 \%$ of diabetic patients. The prescription in hypertensive diabetics with microalbuminuria was lower than in those without microalbuminuria (72\% vs $87 \%, p<0.05)$, but the opposite occurred in pure hypertensive patients with and without microalbuminuria ( $88 \%$ vs $69 \%, p<0.05$ ). There were no significant differences in blood pressure levels, microalbuminuria or serum potassium levels between RAAS blocker users and non-users. No differences were observed either between enalapril and losartan users. Conclusions: The adherence to clinical guidelines is insufficient and users of the recommended drugs did not achieve the expected goals.

(Rev Med Chile 2019; 147: 173-180)

Key words: Diabetes Mellitus; Hypertension; Kidney Failure, Chronic; Renin-Angiotensin System.

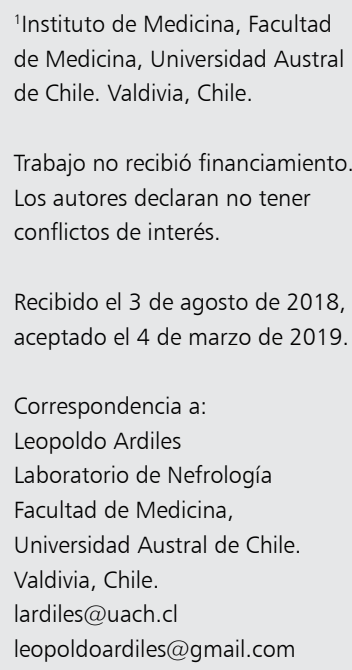

L a enfermedad renal crónica (ERC) representa un problema relevante tanto para el paciente, como para el sistema de salud pública $^{1-4}$. Las guías recomiendan una pesquisa y terapia precoz, a fin de retrasar su progresión ${ }^{5}$ y reducir el riesgo de morbimortalidad cardiovascular asociada ${ }^{6-8}$. Para ello, es necesaria una búsqueda de daño (proteinuria) o falla renal en sujetos de riesgo, para clasificar y orientar adecuadamente su manejo ${ }^{5,9}$.
De este modo, en diabéticos y/o hipertensos proteinúricos, se sugiere el uso de bloqueadores del sistema renina angiotensina aldosterona (SRAA), incluidos los inhibidores de la enzima convertidora de angiotensina (IECA) o los antagonistas de receptores de angiotensina 2 (ARA 2), mientras que en hipertensos sin proteinuria, solo se recomienda un adecuado control de la presión arterial (PA) $)^{5,9-11}$. Ambos fármacos son equivalentes en efectividad antihipertensiva y antiproteinú- 
rica, aunque se diferencian en el menor costo del enalapril ${ }^{12}$ y menos frecuencia de efectos adversos (tos irritativa y reacciones anafilactoídeas) para losartán. A su vez, la principal complicación de ambos es la hiperkalemia, que puede contraindicar o motivar la suspensión de esta terapia ${ }^{5,13,14}$.

El programa de salud cardiovascular (PSCV) de la atención primaria de salud (APS) de Valdivia atiende a sujetos con enfermedad cardiovascular confirmada como hipertensión arterial, diabetes mellitus, dislipidemia, ERC y aquellos en prevención secundaria. El PSCV se adhiere a guías establecidas de pesquisa y manejo de ERC en pacientes con hipertensión arterial (HTA) y diabetes mellitus (DM). No obstante, en la práctica clínica habitual se percibe una subutilización del bloqueo del SRAA, sobre todo en diabéticos proteinúricos, sobre lo que no hay reportes en la literatura nacional.

Se diseñó este estudio para conocer la frecuencia de prescripción del bloqueo del SRAA en pacientes de APS con ERC. Se prefirió seleccionar la etapa 3, considerando que estos pacientes permanecen más tiempo en APS y serían los más beneficiados con la aplicación de las guías clínicas. Nuestra hipótesis plantea un insuficiente cumplimiento de las guías de manejo, con prescripción subóptima de fármacos bloqueadores del SRAA.

\section{Material y Métodos}

Este trabajo fue realizado en el consultorio Jorge Sabat de la comuna de Valdivia, que mostraba una población de 47.341 inscritos al año 2010. Su PSCV se centra en la cobertura y control preferente de pacientes diabéticos e hipertensos y la búsqueda de ERC. Los datos de cada paciente fueron recogidos de su ficha clínica electrónica, y la investigación fue aprobada por el Comité de Ética del Servicio de Salud de Valdivia.

Se revisó los datos de 4.873 pacientes del PSCV activos durante el año calendario 2010, seleccionando a pacientes con diagnósticos de ERC etapa 3 , pues son fácilmente identificables por tener una velocidad de filtración glomerular (VFG) definida $y$ se mantienen en control en APS, con poca intervención de especialistas. Se excluyeron las etapas precoces debido a la dificultad de demostrar daño renal con VFG preservada, como también a aquellos con ERC en etapas avanzadas que se manejan a nivel secundario o terciario.
Para estimar la función renal, se aplicó fórmula MDRD4 ${ }^{15}$ que identificó a sujetos con VFG entre $30 \mathrm{y}<60 \mathrm{ml} / \mathrm{min} / 1,73 \mathrm{~m}^{2}$, como sospechosos de ERC etapa 3. Para cumplir con la definición de ERC, se buscó una segunda creatinina confirmatoria, separada al menos por 3 meses. No se consideraron confirmatorios niveles de creatinina sérica con una variación $>10 \%$ o tomadas en servicios de urgencia, que pueden reflejar una falla renal aguda agregada.

Se recolectaron datos clínicos incluyendo sexo, edad, presión arterial sistólica (PS) y diastólica (PD), relación albuminuria/creatininuria en muestra aislada (RAC) y niveles de potasio. Se consideró bloqueo del eje SRAA cuando los pacientes estuvieran recibiendo enalapril , losartán o ambos, sin perjuicio del uso concomitante de otros fármacos antihipertensivos alternativos a cualquier dosis.

Se definió buen control de presión arterial $<140 / 90 \mathrm{mmHg}$, que es la meta usada en atención primaria ${ }^{16}$.

La RAC se clasificó como normal o meta esperable ( $<30 \mathrm{mg} / \mathrm{g}$ de creatinina), microalbuminuria (30 a $299 \mathrm{mg} / \mathrm{g}$ ) y macroalbuminuria ( $\geq 300 \mathrm{mg} / \mathrm{g}$ ). Hubo pocos sujetos con macroalbuminuria, por lo que se redefinieron solo 2 grupos: RAC normal $(<30 \mathrm{mg} / \mathrm{g})$ y RAC alterado $(\geq 30 \mathrm{mg} / \mathrm{g})$.

Los niveles de potasio se agruparon en: hipokalemia $(<3,5 \mathrm{mEq} / \mathrm{L})$; normokalemia $(3,5 \mathrm{a}$ $5,0 \mathrm{mEq} / \mathrm{L}$ ) e hiperkalemia (>5 mEq/L).

\section{Estadística}

Los resultados de las variables categóricas se expresaron en porcentajes. Para el análisis de variables cualitativas se utilizó tablas de contingencia (Chi cuadrado o test de Fischer). Las variables continuas se expresaron como medianas y rangos y se compararon utilizando Kruskal Wallis y Mann Whitney. Se consideró significación estadística con $\mathrm{p}<0,05$. Se utilizó el software GraphPad InStat 3.01 (Windows 95/NT, San Diego CA, USA).

\section{Resultados}

De los 47.341 inscritos al año 2010, 4.873 estaban adscritos al PSCV (33\% hombres). El tamizaje con la fórmula MDRD4 seleccionó a 491 casos sospechosos de ERC etapa 3.

Una segunda creatinina logró confirmar el diagnóstico en 408 sujetos (Figura 1), constituyen- 
do finalmente $8 \%$ de los sujetos del programa, de los cuales 269 eran mujeres (66\%), con medianas de edad de 77 (51-93) años para hombres y 77 (4098) para mujeres. De ellos, 270 eran hipertensos puros y 138 diabéticos de los cuales 131 (95\%), además, eran hipertensos.

Independiente del diagnóstico, el bloqueo del SRAA se usaba en 303 pacientes (74\%), siendo 159 (52\%) usuarios de enalapril, 137 (45\%) de losartán y 7 (2\%) de terapia dual (ambos medicamentos).

De los 138 diabéticos, $78 \%$ recibía bloqueo del eje y en aquellos que además eran hipertensos, esta prescripción llegaba a 80\%. El 96\% de los diabéticos-hipertensos tenían RAC medido, el que se observaba alterado en $44 \%$ de los casos. El nivel de prescripción de bloqueo en pacientes diabéticos hipertensos con RAC (+) y RAC (-), era de $72 \%$ contra $87 \%$, respectivamente $(\mathrm{p}<0,05)$.

Por su parte, de los 270 hipertensos "puros", $70 \%$ tenía prescrito bloqueo del SRAA. En 211 se disponía de RAC: en ellos, 41 con RAC (+), la prescripción de bloqueo alcanzaba a $88 \%$ y en los 170 con RAC (-), esta llegaba a $69 \%(\mathrm{p}<0,05)$.

No se encontró diferencias en la frecuencia

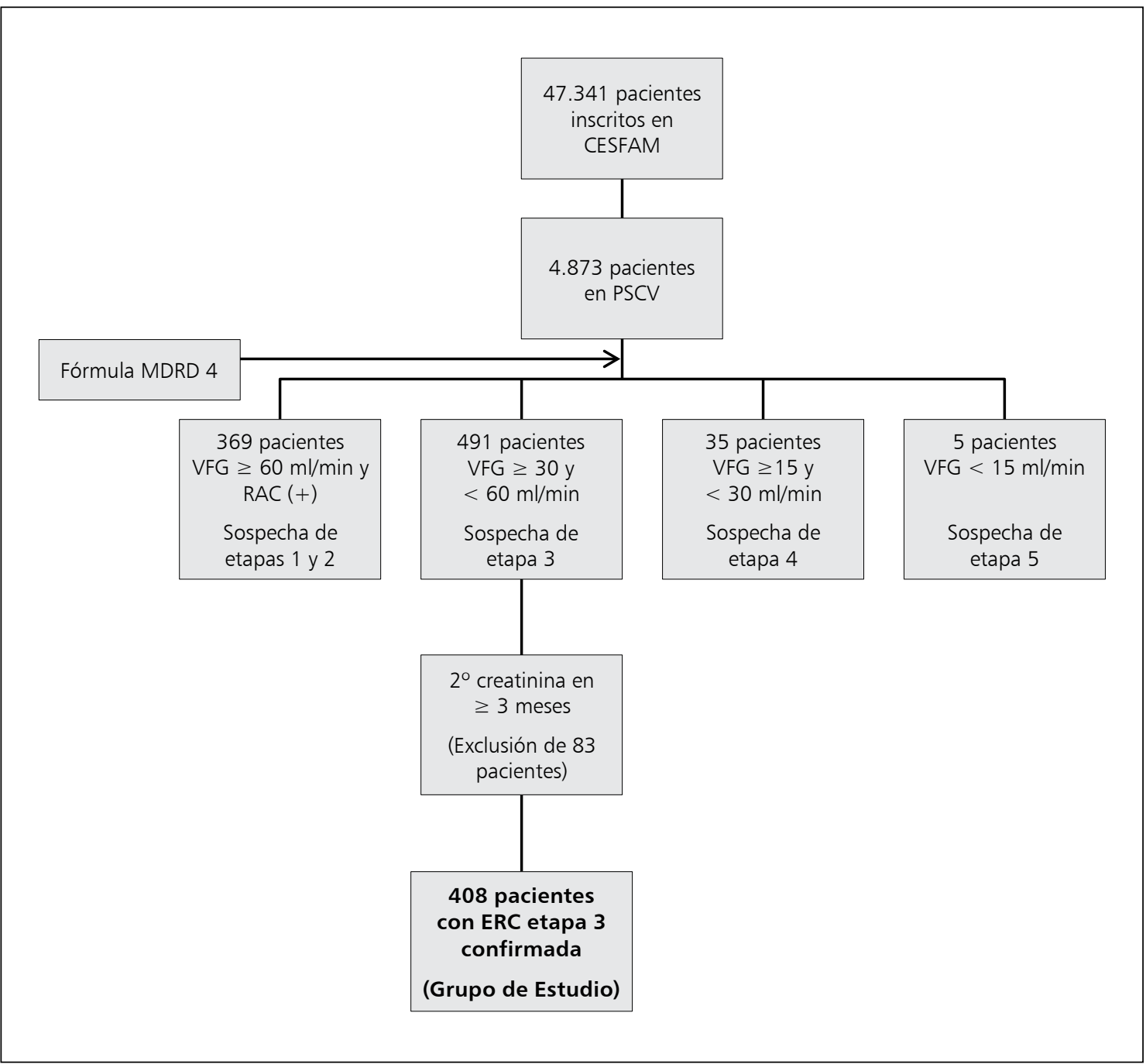

Figura 1. Selección de pacientes con enfermedad renal crónica etapa 3 confirmada. CESFAM: Centro de Salud Familiar; PSCV: programa de salud cardiovascular; MDRD 4: Fórmula abreviada de estimación de la velocidad de filtración glomerular; VFG: velocidad de filtración glomerular estimada; ERC: enfermedad renal crónica; RAC: relación albuminuria/creatininuria (mg/g). 
de control adecuado de la presión arterial entre aquellos que recibían o no bloqueo del eje (39 vs $47 \%$ ), como tampoco entre usuarios de enalapril y losartán (Tabla 1). Tampoco se evidenció diferencias en las medianas de la sistólica (Figura 2) y diastólica (Figura 3) entre usuarios de enalapril, losartán y aquellos sin bloqueo.

También se analizó la frecuencia de sujetos que presentaba RAC normal (Tabla 1), no observando diferencia entre aquellos con o sin bloqueo. En el caso de los diabéticos, las medianas de RAC usando enalapril, losartán o ninguno de ellos, no mostraron diferencias (enalapril 10,6 mg/g; losartán 10,9 mg/g; no bloqueo $48 \mathrm{mg} / \mathrm{g}$ ) (Figura 4).

En 381 pacientes (93\%) se contaba con niveles de potasio. No hubo diferencias al comparar las medianas de las kalemias entre usuarios de enalapril, losartán o ninguno de ellos $(4,8,4,6$ y 4,6 $\mathrm{mEq} / \mathrm{L}$, respectivamente). Noventa (24\%) pre- sentaban algún grado de hiperkalemia, y de ellos, $78 \%$ usaba bloqueo del eje. Hubo 10 pacientes con kalemias $>5,5 \mathrm{mEq} / \mathrm{L}(2,6 \%)$, de los que la mitad tenía bloqueo. Ningún sujeto con bloqueo dual tenía hiperkalemia y no se registró hipokalemia en ningún grupo analizado.

Destacan los resultados encontrados en el pequeño grupo de pacientes con bloqueo dual $(\mathrm{n}=7)$, quienes mostraban presiones sistólicas y diastólicas significativamente mayores, al igual que sus valores de albuminuria (Figuras 2, 3 y 4 ).

Finalmente, se analizó la razón de preferencia para el uso de losartán en lugar de enalapril en 137 casos. No se encontró una razón específica en 95, en 16 se indicó por no lograr buen control tensional con enalapril, en 15 el cambio fue por tos, en 8 se adujo hiperkalemia, en 2 aumento de creatinina y en 1 por persistencia de microalbuminuria.

Tabla 1. Porcentajes de sujetos en buen control de presión arterial (PA) y albuminuria en pacientes con ERC etapa 3, hipertensos y/o diabéticos de un programa de salud cardiovascular en APS, según uso de fármacos bloqueadores del sistema renina angiotensina (SRA)

\begin{tabular}{|c|c|c|c|c|}
\hline & $\begin{array}{c}\text { Sin bloqueo } \\
\text { SRA }\end{array}$ & Enalapril & Losartán & $\begin{array}{c}\text { Bloqueo } \\
\text { dual }\end{array}$ \\
\hline Buen control de PA $(<140 / 90 \mathrm{mmHg})$ & $47 \%$ & $43 \%$ & $34 \%$ & $0 \%$ \\
\hline Buen control de albuminuria ( $<30 \mathrm{mg} / \mathrm{g}$ creatinina) & $74 \%$ & $73 \%$ & $66 \%$ & $14 \%$ \\
\hline
\end{tabular}

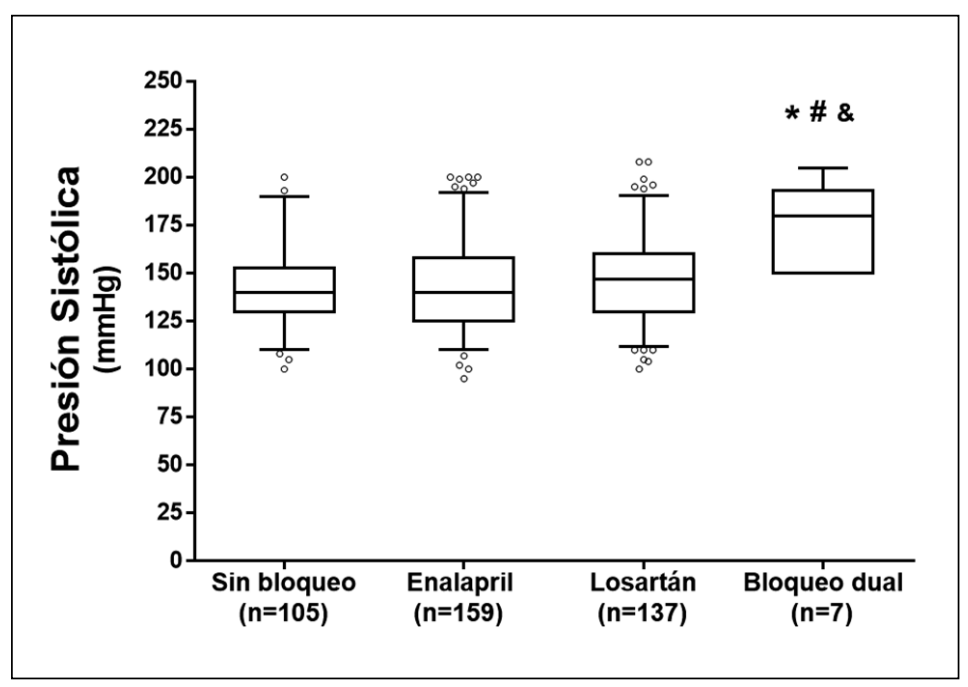

Figura 2. Presión arterial sistólica en pacientes con ERC etapa 3, hipertensos y/o diabéticos de un programa de salud cardiovascular en APS. Las cajas representan el rango intercuartil ( $50 \%$ de los valores); la línea en la caja indica la mediana y los bigotes muestran los percentiles 5 y 95; los círculos identifican los valores extremos. ${ }^{*} p<$ 0,05 comparado con enalapril; ${ }^{*} p<0,05$ comparado con losartán; ${ }^{\&} p<0,05$ comparado con grupo sin bloqueo. 

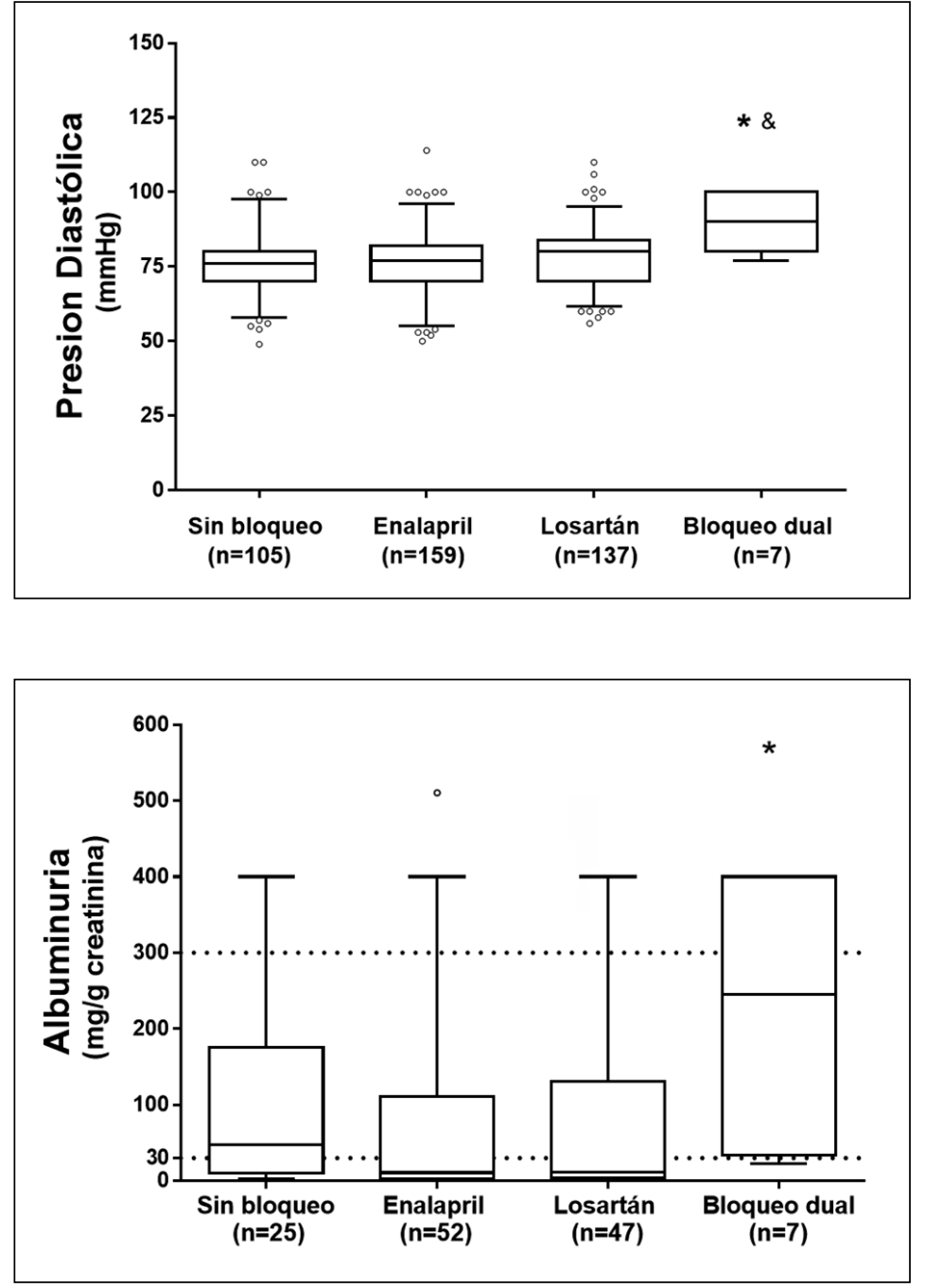

Figura 3. Presión arterial diastólica en pacientes con ERC etapa 3, hipertensos y/o diabéticos de un programa de salud cardiovascular en APS. Las cajas representan el rango intercuartil ( $50 \%$ de los valores); la línea en la caja indica la mediana y los bigotes muestran los percentiles 5 y 95; los círculos identifican los valores extremos. * $p$ $<0,05$ comparado con enalapril; ${ }^{\&} p<0,05$ comparado con grupo sin bloqueo.

Figura 4. Albuminuria en pacientes con ERC etapa 3, hipertensos y/o diabéticos de un programa de salud cardiovascular en APS. Las cajas representan el rango intercuartil ( $50 \%$ de los valores); la línea en la caja indica la mediana y los bigotes muestran los percentiles 5 y 95; los círculos identifican los valores extremos. Las líneas punteadas sobre el fondo del gráfico representan los valores de albuminuria entre 30-300 mg/g creatinina. ${ }^{*} p<0,05$ comparado con enalapril.

\section{Discusión}

Este trabajo analiza la prescripción de bloqueo de SRAA en pacientes con ERC como modelo de adherencia a protocolos del PSCV.

La población estudiada resultó ser añosa, concordante con la literatura revisada ${ }^{2,17-19}$, reflejando el envejecimiento poblacional y el que los jóvenes priorizan poco el control de patologías asintomáticas. Hay un claro predominio de población femenina, algo reportado en publicaciones nacionales ${ }^{19,20}$, pero que no tiene antecedentes en la literatura internacional ${ }^{2,17,18}$. Las razones de esta preponderancia no quedan claras, aunque pueden deberse a la menor inserción laboral de la mujer y controles ginecoobstétricos que facilitan la pesquisa precoz de otras patologías.

Del total de inscritos en el consultorio, solo $10 \%$ estaba adscrito al PSCV, muy por debajo de lo esperado de acuerdo a la Encuesta Nacional de Salud chilena 2010 (ENS) ${ }^{21}$. En efecto, la ENS establece prevalencias de HTA y DM de 26,9\% y $9,4 \%$ respectivamente. Sin embargo, la misma ENS menciona que solo $32,2 \%$ de los hipertensos y $52 \%$ de los diabéticos se encuentran bajo tratamiento, cifras que son más coincidentes con este consultorio y que reflejan una baja cobertura como problemática nacional. 
El análisis de nuestro objetivo principal -prescripción de bloqueo del SRAA en ERC 3- resultó difícil, ya que existe escasa literatura al respecto y los estudios se suelen centrar en los beneficios de IECA o ARA 2 y no en la prescripción misma. Un estudio internacional en población diabética mostró una prescripción de IECA o ARA 2 en $53,9 \%$ de los casos, y en diabéticos hipertensos con y sin ERC alcanzó a 85 y $71 \%$, respectivamente 22 . En nuestra muestra hubo una proporción de uso similar (74\%), quedando como tarea importante conocer la causa de la omisión en uno de cada 4 sujetos.

En ese sentido, en los diabéticos proteinúricos hay más evidencia bibliográfica del beneficio del bloqueo del eje, tanto en la prevención de progresión de la enfermedad renal como en la reducción del riesgo cardiovascular ${ }^{5,9,11}$. En nuestro grupo, la indicación alcanzaba a 78\% del total de diabéticos, cifra que sube a $80 \%$ en diabéticos con HTA. Sin embargo, cuando se comparó diabéticos hipertensos con y sin proteinuria, esta prescripción fue significativamente menor en aquellos con proteinuria, lo que puede sugerir una mala interpretación de la norma buscando solo el control tensional.

En el caso de hipertensos puros, la evidencia y las guías clínicas recomiendan el control de PA con cualquier fármaco ${ }^{5,9,10}$. Aun así, las mismas guías de HTA del Ministerio de Salud proponen el uso del bloqueo del SRAA o $\beta$ bloqueadores como terapia de primera línea en $<55$ años ${ }^{16}$. El uso de IECA o ARA 2, plenamente validado en pacientes hipertensos proteinúricos ${ }^{5,9,10}$, en nuestro estudio mostró una mayor indicación en los hipertensos proteinúricos que en aquellos no proteinúricos, reflejando probablemente una mayor intención de tratamiento específico, algo que habríamos deseado para el caso de los diabéticos.

Curiosamente, destaca el hecho que $78 \%$ de los hipertensos puros cuenten con RAC vigente, considerado que este examen no está incluido aún como rutina exploratoria de hipertensos en nuestro país ${ }^{5,16}$. Es posible que se solicite por la caída de la VFG y no por la HTA. Como sea, 19\% tenían RAC (+), representando al menos un grupo de mayor riesgo CV o quizá a pacientes con una nefropatía subyacente, cuya expresión es la HTA y proteinuria; lamentablemente, no detectamos un afán exploratorio mayor en estos sujetos.
La literatura avala el uso de IECA o ARA 2 como equivalentes para el control de PA y microalbuminuria ${ }^{5,9-11,16}$. Llama profundamente la atención la ausencia de diferencias significativas en el grado de alcance de metas de presión y RAC, así como en sus valores absolutos, entre los pacientes sin bloqueo del eje y aquellos que recibían enalapril o losartán, lo que puede estar sugiriendo un uso inadecuado de dosis o problemas de adherencia. Lamentablemente, nuestros datos, que carecen de información al respecto, no permiten aclarar esta última duda.

Otro punto importante son los niveles de potasio, toda vez que la hiperkalemia es efecto adverso y contraindicación frecuente en estas terapias $^{5,13,14,23}$. Nuestro estudio demostró que casi un cuarto de los pacientes tenía hiperkalemia, aunque solo $2,6 \%$ tiene niveles de potasio $>5,5 \mathrm{mEq} / \mathrm{L}$ y solo en 1 caso se observó kalemia $>6 \mathrm{mEq} / \mathrm{L}$, ninguno de ellos bajo terapia dual. Esto refuerza la idea que pocos sujetos con ERC en etapa 3, donde está más indicada esta terapia, tienen contraindicación para su uso.

Por su parte, la prescripción de losartán alcanzó a $45 \%$ de los sujetos, lo que resulta llamativo, considerando que es 2 veces más caro que enalapril, lo que a nivel institucional puede resultar relevante, por lo que su uso se justificaría solo ante tos inducida por enalapril, que se presenta hasta en $12 \%$ de los paciente ${ }^{24,25}$. Nuestro estudio concluyó que $11 \%$ de los usuarios de enalapril fueron cambiados a losartán por tos, por lo que se esperaría un uso más limitado. Sin embargo, en $20 \%$ de los usuarios de losartán se adujeron razones farmacológicamente incorrectas para preferirlo, como intentar mejor control de PA o microalbuminuria, evitar hiperkalemia y, en casi $70 \%$ de los casos, sin identificarse alguna razón específica. En definitiva, la indicación de losartán es farmacológicamente apropiada, pero no siempre tiene un sustento clínico justificado. Las razones de su preferencia aparecen inciertas y se podría pensar en una intención de probar un fármaco de incorporación más reciente o considerarlo erróneamente más potente para el control de la PA y microalbuminuria.

Adicionalmente, resulta de interés que 7 casos usaban bloqueo dual (enalapril más losartán), siendo todos hipertensos, 6 proteinúricos y solo 2 diabéticos. Solo uno de ellos correspondía a un hipertenso sin microalbuminuria, siendo los 6 
restantes sujetos proteinúricos en los que podemos elucubrar que correspondían a nefropatías más graves o simplemente peor logro de las metas. Como sea, la indicación de bloqueo dual es una estrategia que requiere intervención de subespecialistas y no forma parte de las recomendaciones de manejo en atención primaria, menos aun con una intención puramente antihipertensiva ${ }^{5}$.

Aunque nuestro estudio adolece de la evaluación de otros aspectos como el control glicémico, lipídico y el uso de otros fármacos, evidencia una adherencia incompleta a un aspecto destacado de las recomendaciones de las guías clínicas. Tampoco incluyó las dosis de enalapril o losartán utilizadas que permitieran una mejor interpretación de sus efectos.

La ERC es una patología de gran relevancia en APS y pese a las recomendaciones, la prescripción de bloqueo del eje aún resulta insuficiente, especialmente en grupos de riesgo.

Nuestro trabajo permite reconocer las dificultades de aplicación de las guías clínicas y sugiere la necesidad de un apoyo educativo más cercano de parte de los subespecialistas a este nivel de atención.

Reconocimientos: Este trabajo formó parte de la tesis del Dr. Marcel Álvarez en la obtención del Magíster en Metodologías Clínicas y Epidemiológicas para la Práctica Médica de la Escuela de Graduados de la Facultad de Medicina, Universidad Austral de Chile.

\section{Referencias}

1. Evans PD, Taal MW. Epidemiology and causes of chronic kidney disease. Medicine 2015; 43 (8): 450-3.

2. Zhang QL, Rothenbacher D. Prevalence of chronic kidney disease in population-based studies: systematic review. BMC Public Health 2008; 8: 117.

3. Ryan TP, Sloand JA, Winters PC, Corsetti JP, Fisher SG. Chronic kidney disease prevalence and rate of diagnosis. Am J Med 2007; 120 (11): 981-6.

4. Jha V, García-García G, Iseki K, Li Z, Naicker S, Plattner B, et al. Chronic kidney disease: global dimension and perspectives. Lancet 2013; 382 (9888): 260-72.

5. Ministerio de Salud, Gobierno de Chile. Guia Clínica: Prevención de enfermedad renal crónica. Minsal 2010.

6. Pugliese G, Solini A, Bonora E, Fondelli C, Orsi E, Nicolucci A, et al. Chronic kidney disease in type 2 diabetes: lessons from the Renal Insufficiency And Cardiovascular Events (RIACE) Italian Multicentre Study. Nutr Metab Cardiovasc Dis 2014; 24 (8): 815-22.

7. Wattanakit K, Coresh J, Muntner P, Marsh J, Folsom AR. Cardiovascular risk among adults with chronic kidney disease, with or without prior myocardial infarction. J Am Coll Cardiol 2006; 48 (6): 1183-9.

8. Ozyol A, Yucel O, Ege MR, Zorlu A, Yilmaz MB. Microalbuminuria is associated with the severity of coronary artery disease independently of other cardiovascular risk factors. Angiology 2012; 63 (6): 457-60.

9. Tomson C, Bailey P. Management of chronic kidney disease. Medicine 2011; 39 (7): 407-13.

10. Ruzicka M, Burns KD, Culleton B, Tobe SW, Canadian Hypertension Education P. Treatment of hypertension in patients with nondiabetic chronic kidney disease. Can J Cardiol 2007; 23 (7): 595-601.

11. Jaber BL, Madias NE. Progression of chronic kidney disease: can it be prevented or arrested? Am J Med 2005; 118 (12): 1323-30.

12. CENABAST (https://www.cenabast.cl/wp-content/ uploads/2017/11/Informe-Web-Octubre-2018.pdf) (Acceso el 10 de Noviembre 2018).

13. Jain N, Kotla S, Little BB, Weideman RA, Brilakis ES, Reilly RF, et al. Predictors of hyperkalemia and death in patients with cardiac and renal disease. Am J Cardiol 2012; 109 (10): 1510-3.

14. Kurnik D, Vesterman-Landes J, Bialik M, Katzir I, Lomnicky Y, Halkin H, et al. Hyperkalemia and renal function during monotherapy and dual renin-angiotensin blockade in the community setting. Clin Ther 2011; 33 (4): 456-64.

15. Levey AS, Eckardt KU, Tsukamoto Y, Levin A, Coresh J, Rossert J, et al. Definition and classification of chronic kidney disease: a position statement from Kidney Disease: Improving Global Outcomes (KDIGO). Kidney Int 2005; 67 (6): 2089-100.

16. Ministerio de Salud, Gobierno de Chile. Guía clínica: Hipertensión arterial primaria o esencial en personas de 15 años y más. Minsal 2010.

17. Lou Arnal LM, Campos Gutiérrez B, Cuberes Izquierdo M, Gracia García O, Turon Alcaine JM, Bielsa García S, et al. Prevalence of chronic kidney disease in patients with type 2 diabetes mellitus treated in primary care. Nefrologia 2010; 30 (5): 552-6.

18. Martínez-Castelao A, Gorriz JL, Portoles JM, De Alvaro F, Cases A, Luno J, et al. Baseline characteristics of patients with chronic kidney disease stage 3 and stage 4 in Spain: the MERENA observational cohort study. BMC Nephrol 2011; 12: 53.

19. Zúñiga SMC, Muller OH, Flores OM. [Prevalence of 
chronic kidney disease in subjects consulting in urban primary care clinics]. Rev Med Chile 2011; 139 (9): 1176-84.

20. Villarroel RP, Parra LX, Ardiles AL. [Frequency of chronic kidney disease among ambulatory patients with type 2 diabetes]. Rev Med Chile 2012; 140 (3): 287-94.

21. Ministerio de Salud, Gobierno de Chile. Encuesta Nacional de Salud ENS Chile 2009-2010.

22. Cooke CE, Fatodu H. Physician conformity and patient adherence to ACE inhibitors and ARBs in patients with diabetes, with and without renal disease and hypertension, in a medicaid managed care organization. JMCP 2006; 12 (8): 649-55.
23. Sadjadi SA, McMillan JI, Jaipaul N, Blakely P, Hline SS. A comparative study of the prevalence of hyperkalemia with the use of angiotensin-converting enzyme inhibitors versus angiotensin receptor blockers. Ther Clin Risk Manag 2009; 5 (3): 547-52.

24. Bangalore S, Kumar S, Messerli FH. Angiotensin-converting enzyme inhibitor associated cough: deceptive information from the Physicians' Desk Reference. Am J Med 2010; 123 (11): 1016-30.

25. Mancia G, Schumacher H. Incidence of adverse events with telmisartan compared with ACE inhibitors: evidence from a pooled analysis of clinical trials. Patient Prefer Adherence 2012; 6: 1-9. 